FACTA UNIVERSITATIS (NIŠ)

Ser. Math. Inform. Vol. 34, No 4 (2019), 671-678

https://doi.org/10.22190/FUMI1904671R

\title{
PARTITION-EQUIVALENT n-POINTS CONFIGURATIONS WITH TWO DISTANCES *
}

\author{
Ali Asghar Rezaei
}

(C) 2019 by University of Niš, Serbia | Creative Commons Licence: CC BY-NC-ND

Abstract. In this paper we define an equivalence relation on the set of all possible geometrical models of $M(n, k)$ containing $\mathrm{n}$ points in 3D Euclidean space having $k$ distinct distances. We investigate the number of geometrical models for $M(4,2), M(5,2)$ and $M(6,2)$ up to the mentioned equivalence relation.

Keywords. Constructible models; distinct distances; partition-equivalent; geometrical model.

\section{Introduction}

Distance geometry has considered two main problems since its inception. One of these problems is the study of the embedding of a semimetric space in an Euclidean space. From the empirical point of view, $\mathbb{R}^{3}$ is the most important Euclidean space, especially in several applications such as molecular conformation, wireless sensor networks, statics, dimensionality reduction, and robotics. In these applications input data is a set of points and their pair-wise distances(a semimetric space) and the output is a set of points in $\mathbb{R}^{3}$ realizing those given distances.

Our task is to focus on the number of distinct distances in a semimetric space. A semimetric space with $n$ points and $k$ distinct distances may be embedable in $\mathbb{R}^{3}$ or not. Such space is denoted by $M(n, k)$ and if it can be embedded in $\mathbb{R}^{3}$, we say that $M(n, k)$ is constructible. Such problems have been extensively researched, and yet in many cases are still wide open (see for example $[4,3,7]$ ). Some computational theorems have been proved in [5] for $M(n, k)$. In [6] an equivalence relation was introduced for all models of $M(n, k)$ and the author classified all possible models for $M(4,2)$ and $M(5,2)$. In this paper we define a new equivalence relation in term

Received March 02, 2019; accepted May 11, 2019

2010 Mathematics Subject Classification. Primary 51D20; Secondary 05B25, 05B30

*The author was supported in part by University of Kashan 
of the partitions of a natural number and find the number of the equivalence classes for $M(4,2), M(5,2)$, and $M(6,2)$.

The paper has been organized as follow: First we provide some preliminaries and definitions. Using the partition of a number, we then define an equivalence relation to classify the models of $M(n, k)$. Finally we will investigate $M(6,2)$ in term of the mentioned equivalence relation.

\section{Definitions and Notations}

In this section we introduce the basic concepts which are used through the paper. Some of these preliminaries have been defined in $[1,6,5]$.

Definition 2.1. A semimetric on a set $S$ is a function $d: S \times S \rightarrow[0, \infty)$ which satisfies the following properties:

- $d(x, y)=d(y, x)$ for all $x, y \in S$.

- $d(x, y)=0$ if and only if $x=y$.

A semimetric space is a pair $(S, d)$ where $S$ is a set and $d$ is a semimetric on it.

When $d$ is understood, we usually omit mention of it and just say " $S$ is a semimetric space." In some literature $d$ is called the distance function. The distance between two points $p$ and $q$ is denoted in both notations $d(p, q)$ or $p q$.

The problem of embedding an arbitrary semimetric space isometrically into $\mathbb{R}^{3}$ is an interesting task in Distance Geometry. A necessary condition for embedding can be stated in term of Cayley-Menger determinant.

Definition 2.2. Let $\left\{p_{0}, p_{1}, \ldots, p_{k}\right\}$ be a semimetric space. The Cayley-Menger determinant for this $k+1$-tuple is defined as

$$
D\left(p_{0}, \ldots, p_{k}\right)=\left|\begin{array}{ccccc}
0 & 1 & 1 & \ldots & 1 \\
1 & 0 & p_{0} p_{1}^{2} & \ldots & p_{0} p_{k}^{2} \\
\ldots & \ldots & \ldots & \ldots & \ldots \\
1 & p_{k} p_{0}^{2} & p_{k} p_{1}^{2} & \ldots & 0
\end{array}\right|
$$

Theorem 2.1. [1] A necessary condition that a semimetric $r+1$-tuple $\left\{p_{0}, p_{1}, \ldots, p_{r}\right\}$ be isometrically embedded in an Euclidean space $\mathbb{R}^{n}$ is that for every $k=1,2, \ldots, r$ the determinant $D\left(p_{0}, \ldots, p_{k}\right)$ either vanish or have the sign of $(-1)^{k+1}$. If $n<r$, then $D\left(p_{0}, \ldots, p_{k}\right)=0(n<k \leqslant r)$.

We will consider this theorem for $n=3$ and $r=5$. Note that if a five-points semimetric space $\left\{p_{0}, \ldots, p_{4}\right\}$ can an embedded in $\mathbb{R}^{3}$, then a necessary condition for embedding six points $\left\{p_{0}, p_{1}, \ldots, p_{5}\right\}$ in $\mathbb{R}^{3}$ is that $D\left(p_{0}, \ldots, p_{5}\right)=0$. 
Definition 2.3. Let $S=\left\{p_{1}, p_{2}, \ldots, p_{n}\right\}$ be a semimetric space such that

$$
\operatorname{card}\left\{d\left(p_{i}, p_{j}\right) \mid i \neq j, i, j=1,2, \ldots, n\right\}=k
$$

( $d$ is the distance function). Then $S$ is called a model with $n$ points and $k$ distances and is denoted by $M(n, k)$. If $S$ can be isometrically embedded in $\mathbb{R}^{3}$, then we say $M(n, k)$ is constructible .

For example $M(5,1)$ is not constractible, while $M(4,1)$ is constructible. In [6] $M(n, 2)$ was investigated for $n \leqslant 5$. Here we consider the case $n=6$. Note that $M(n, 2)$ is not constructible for $n>6[2]$.

Definition 2.4. [5] Let $m, m_{1}, m_{2}, \ldots, m_{k}$ are natural numbers such that

$$
m=m_{1}+m_{2}+\cdots+m_{k}, \quad 1 \leqslant m_{1} \leqslant m_{2} \leqslant \ldots \leqslant m_{k} .
$$

Then the summand $m_{1}+m_{2}+\cdots+m_{k}$ is called a $k$-partition for $m$.

For example $1+9,2+8,3+7,4+6$, and $5+5$ are 2-partitions for 10. Similarly, 2partitions for 15 are $1+14,2+13$, and so on.

Notation. We correspond to each model $M(n, k)$, a $k$-partition of $m=n(n-1) / 2$ (the number of edges) as follow. Let $d_{1}, d_{2}, \ldots, d_{k}$ be the distances in this model and $m_{j}$ be the number of edge with length $d_{j}$. Without loss of generality we can assume $m_{1} \leqslant m_{2} \leqslant \ldots \leqslant m_{k}$. Then the number of all edges is

$$
m=m_{1}+m_{2}+\cdots+m_{k} .
$$

We also correspond to each model $M(n, k)$, a colored graph with $n$ vertices in which the edges with same length have same color.

Definition 2.5. Two models for $M(n, k)$ are said to be partition-equivalent if their $k$-partitions are same.

For example the following models for $M(5,2)$ are partition-equivalent with 2-partitions $4+6$ :

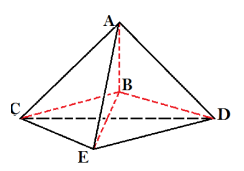

(a)

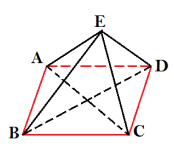

(b)

In (a), the points $\mathrm{A}, \mathrm{C}, \mathrm{E}$, and $\mathrm{D}$ are vertices of a regular tetrahedron and $\mathrm{B}$ is its center, while in (b), the points A, B, C, D, and E are the vertices of a right pyramid whose base is a unit squar with edge length equal to $\sqrt{2}$.

One can easily show that partition-equivalence is an equivalence relation on the set of all constructible models for $M(n, k)$.

It was shown that all 2-partitions of 10 concerning to $M(5,2)$ are constructible [6]. So up to partition-equivalence there are exactly 5 geometrical models for $M(5,2)$. Similarly all 2-partitions of 6 concerning to $M(4,2)$ are constructible, so there are exactly 3 geometrical model for $M(4,2)$ up to partition-equivalence. In the next section we complete our task to classify all models for $M(n, 2)$ up to partition-equivalence by considering $M(6,2)$. 


\section{Partition-Equivalent Models for $M(6,2)$}

For 6 points in $\mathbb{R}^{3}$ the number of edges is

$$
\left(\begin{array}{l}
6 \\
2
\end{array}\right)=15 .
$$

As we see before, there are seven 2-partitions for 15: 1+14, 2+13, 3+12, 4+11, $5+10,6+9$, and $7+8$. So one can say that there are at most seven constructible model for $M(6,2)$ up to partition-equivalence. But as we will see some of these partitions are not constructible. Our goal is to determine which of these partitions are constructible. The construction is as follow: starting with all possible graphs for each partition $m+n=15$, we next investigate which graph is constructible. In the rest of paper we use the notation $P(m, n)$ for the partition $m+n=15$.

First we show that $P(3,12), P(5,10)$, and $P(6,9)$ are constructible.

Proposition 3.1. $P(3,12)$ is constructible for $M(6,2)$.

Proof. Let the points A, B, C, D, E, and F are the vertices of a regular octahedron as follows:

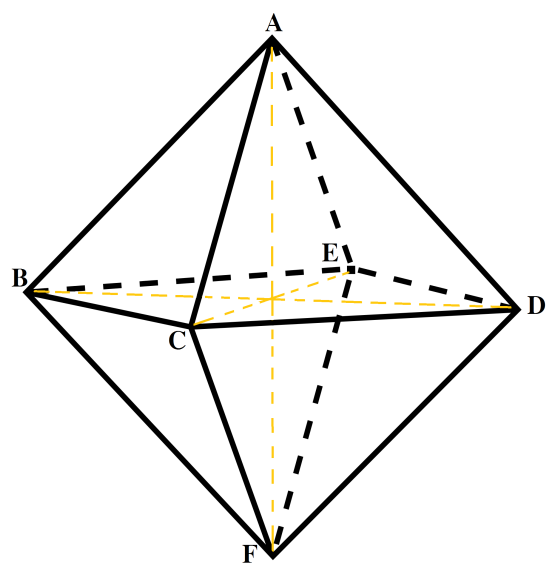

If we take $d(A, B)=1$ as other edges, then $d(B, D)=d(C, E)=d(A, F)=\sqrt{2}$. In fact this shape is the geometrical realization for the following graph whose 2partition is $P(3,12)$.

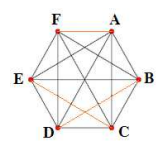

This completes our argument.

Note that for $P(3,12)$ we have some other graphs. But to be constructible, it is sufficient to find one graph having a geometric realization as above.

Proposition 3.2. $P(5,10)$ is constructible for $M(6,2)$. 
Proof. Take the points A, B, C, D, E, and F as the vertices of a regular pyramid whose base is a regular pentagon as follow.

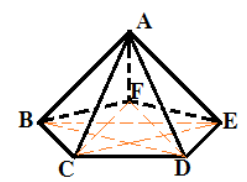

If we assume for example $d(A, B)=1$ (and the same for other edges), then $d(B, D)=$ $d(C, E)=d(A, D)=d(B, E)=d(A, C)=\sqrt{2-2 \cos 3 \pi / 5}$. So this is a geometrical realization for the foolowing graph of $P(5,10)$.

This completes our argument.

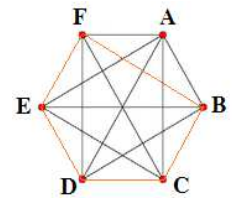

Proposition 3.3. $P(6,9)$ is constructible for $M(6,2)$

Proof. Let A, B, C, D, E, and F are the vertices of a right prism as follow:

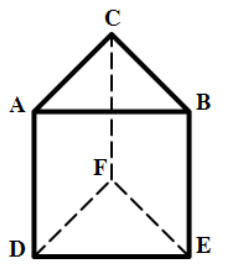

Take $d(A, B)=1$ (and the same for other edges), then $d(B, D)=d(A, E)=$ $d(A, D)=d(B, F)=d(E, C)=d(A, F)=d(C, D)=\sqrt{2}$. The above shape is a realization for the following graph of $P(6,9)$.

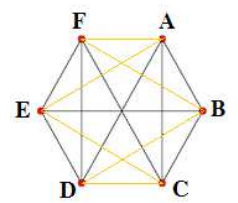

This completes our argument.

Now we continue with non-constructible partitions.

Proposition 3.4. $P(1,14)$ is not constructible for $M(6,2)$.

Proof. The corresponding graph for $P(1,14)$ is as follow.

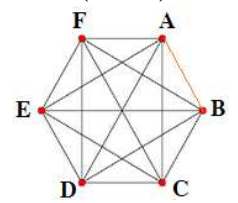


If this graph have a geometric realization, then the five points $A, C, D, E, F$ have equal pair-wise distances, which is impossible, because in $\mathbb{R}^{3}$ there are at most four points with this property.

Proposition 3.5. $P(2,13)$ is not constructible for $M(6,2)$

Proof. For $P(2,13)$ there are two non-isomorphic graphs as follows:
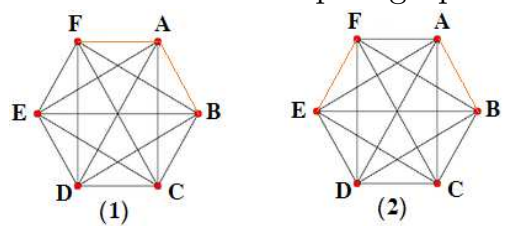

Graph (1) has no geometrical realization, because it is impossible that five points $\mathrm{B}, \mathrm{C}, \mathrm{D}, \mathrm{E}$, and $\mathrm{F}$ have same pair-wise distances.

We show the same statement for graph (2). If graph (2) has a geometrical realization then the points $\mathrm{B}, \mathrm{C}, \mathrm{D}$, and $\mathrm{E}$ are vertices of a regular tetrahedron and so are $\mathrm{F}, \mathrm{D}, \mathrm{C}$, and $\mathrm{B}$.These two pyramids have the common triangle $\mathrm{BCD}$ as a common face and hence the only possible geometric structure for these five points is as follows:

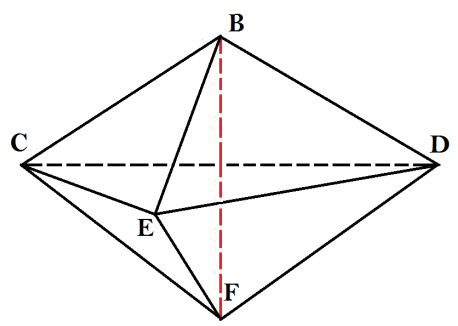

If we assume $d(B, D)=1$, then $d(B, F)=2 \sqrt{2 / 3}$. It means that $d(B, D) \neq$ $d(B, F)$, while these two edges in graph (2) have same length. So the graph (2) has no geometrical realization.

The argument used in the above proposition will be used in next proposition. We will recall this argument as two regular pyramids with a common face.

Proposition 3.6. $P(4,11)$ is not constructible for $M(6,2)$.

Proof. All possible graphs for $P(4,11)$ have been presented in the following figure: 

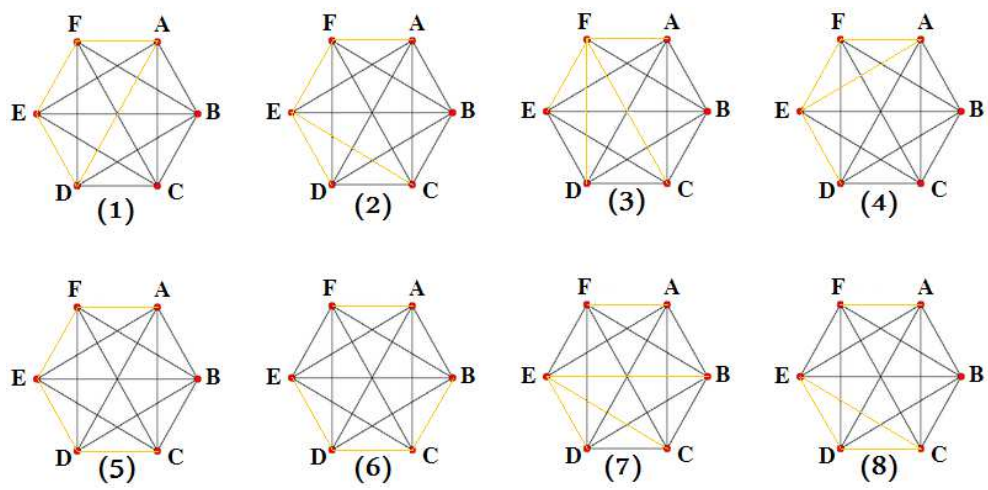

We show that these graphs have no realizations in $\mathbb{R}^{3}$. In graphs (2), (4), and (7) we have two regular pyramids with a common face. Due to the length of the other edges, it follows that these graphs have no realization in $\mathbb{R}^{3}$.

Graphs (3) is not constructible since the points $A, B, C, D$, and $E$ have same pair-wise distances which is impossible in $\mathbb{R}^{3}$.

Now consider the graph (1). We have two regular pyramids $A B C E$ and $B C D F$ with common edge $B C$. Since $E D=A F$, the position of two pyramids is symmetric. Without lose of generality to construct these pyramids one can take the vertices as follows:

$$
\begin{gathered}
A=\left(\frac{1}{2}, 0, \frac{\sqrt{2}}{2}\right), \quad B=\left(0,-\frac{1}{2}, 0\right), \quad C=\left(0, \frac{1}{2}, 0\right), \\
D=\left(-\frac{1}{2}, 0,-\frac{\sqrt{2}}{2}\right), \quad E=\left(-\frac{1}{2}, 0, \frac{\sqrt{2}}{2}\right), \quad F=\left(\frac{1}{2}, 0,-\frac{\sqrt{2}}{2}\right) .
\end{gathered}
$$

By simple calculation one can see that $A F=E D=\sqrt{2}$ and $A D=\sqrt{3 / 2}$, so $A D \neq A F$, while in (1) we have $A D=A F$.

For the remaining graphs, we use Theorem 2.1. First consider the graph (5). Omit the point $E$ for a moment, the remaining points $A, B, C, D$, and $F$ are vertices of a right pyramid whose base is a square of side 1 ( $B$ is the apex of pyramid), so $A F=D C=\sqrt{2}$. If this garph has a realization in $\mathbb{R}^{3}$, then its Cayley-Menger determinant must be zero. But we have

$$
D(A, B, C, D, E, F)=\left|\begin{array}{lllllll}
0 & 1 & 1 & 1 & 1 & 1 & 1 \\
1 & 0 & 1 & 1 & 1 & 1 & 2 \\
1 & 1 & 0 & 1 & 1 & 1 & 1 \\
1 & 1 & 1 & 2 & 0 & 2 & 1 \\
1 & 1 & 1 & 1 & 2 & 0 & 2 \\
1 & 2 & 1 & 1 & 1 & 2 & 0
\end{array}\right|=5 \neq 0 .
$$

Same argument can be applied for the graphs (6) and (8) by disregarding the point $D$. It is easy to see that $D(A, B, C, D, E, F)=-4$ for graph (6), and $D(A, B, C, D, E, F)=-16$ for graph (8). So the necessary condition in Theorem 2.1 does not hold for these cases. 
The only partition which has not been specified is $P(7,8)$. Because of its variety, the investigation of $P(7,8)$ requires a separate research (there are at least 19 nonisomorphic graphs for $P(7,8))$. The author's research for $P(7,8)$ has been led to the following conjecture:

Conjecture 3.1. $P(7,8)$ is not constructible for $M(6,2)$.

Regardless of whether the above conjecture is correct or not we have already proved the following important theorem:

Theorem 3.1. Up to partition-equivalence, there are at least 3 constructible models for $M(6,2)$.

Acknohledgement. The author is grateful to the University of Kashan for supporting this work by Grant No. 682421/1.

\title{
R E F E R E N C E S
}

1. L. Blumenthal: Theory and Applications of Distance Geometry. Oxford University Press, Oxford, 1953.

2. H. T. Croft: 9-point and 7-point configurations in 3-space. Proc. London Math. Soc. 12 (1962), 400-424.

3. G. Elekes and M. ShariR: Incidences in three dimensions and distinct distances in the plane. Combin. Probab. Comput. 20 (2011), 571-608.

4. L. Guth and N. H. Katz: On the Erdös distinct distances problem in the plane. Ann. of Math. (2015), 155-190.

5. A. A. REZAEI: On the geometric structures with $n$ points and $k$ distances. Electron. Notes Discrete Math. 45 (2014), 181-186.

6. A. A. Rezaei: On the Configurations with $n$ Points and Two Distances. Math. Int. Res. DOI: $10.22052 /$ mir.2017.81496.1056

7. G. Tardos: On distinct sums and distinct distances. Adv. Math. 180 (2003), 275-289.

\author{
Ali Asghar Rezaei \\ Faculty of Mathematical Sciences \\ Department of Pure Mathematics \\ University of Kashan \\ Kashan, Iran \\ a_rezaei@kashanu.ac.ir
}

